\title{
CONTRIBUTION TO THE SOLUTION OF THE COUETTE INVERSE PROBLEM FOR HERSCHEL-BULKLEY FLUIDS BY MEANS OF THE INTEGRATION METHOD
}

\author{
$\underline{\text { Gert Heirman }}^{(1)}$, Lucie Vandewalle ${ }^{(1)}$, Dionys Van Gemert ${ }^{(1)}$, Ólafur Wallevik ${ }^{(2)}$ and \\ $\overline{\text { Niki Cauberg }}^{(3)}$
}

(1) Department of Civil Engineering - Reyntjens Laboratory, K.U.Leuven, Belgium

(2) Icelandic Building Research Institute (IBRI) - Rheocenter, Reykjavík, Iceland

(3) Belgian Building Research Institute (BBRI), Belgium

\begin{abstract}
For Belgian SCC mixes, based on the powder-type philosophy of SCC mix design, a Herschel-Bulkley (HB) behaviour of the fresh SCC mixes is quite often observed.

A longstanding problem in rheometry is the so-called "Couette inverse problem", in which one tries to derive the flow curve $\tau(\dot{\gamma})$ from the torque measurements $\mathrm{T}(\mathrm{N})$ in a (wide-gap) coaxial cylinder (Couette) rheometer, where $\mathrm{T}$ represents the torque registered at the inner, stationary, cylinder and $\mathrm{N}$ is the rotational velocity of the outer, rotating, cylinder.

In this paper, the integration method is studied in order to find out if an analytical equation can be found for converting $\mathrm{T}(\mathrm{N})$ into $\tau(\dot{\gamma})$. For a Bingham fluid, this conversion equation is known as the "Reiner-Riwlin" equation.

In the first part of the paper, the underlying physics and the derivation of the Reiner-Riwlin equation are given by means of introduction for the Herschel-Bulkley fluid. In the second part of the paper, the mathematical consequences of the derivation of the governing conversion equation for Herschel-Bulkley fluids are described.
\end{abstract}

Keywords: Rheology, self-compacting concrete, Couette inverse problem, Bingham fluid, Herschel-Bulkley fluid. 


\begin{tabular}{|c|c|c|c|}
\hline \multirow{2}{*}{\multicolumn{2}{|c|}{$\begin{array}{l}\text { Nomenclature } \\
\text { Latin characters }\end{array}$}} & \multirow{3}{*}{$\begin{array}{l}\hat{\tau}_{0} \\
\Omega_{0}\end{array}$} & \multirow{3}{*}{$\begin{array}{l}\text { yield value for B fluids [Pa] (eq. } 2 \text { ) } \\
\text { angular velocity of the outer cylinder } \\
{[\mathrm{rad} / \mathrm{s}](\equiv 2 \pi \mathrm{N})}\end{array}$} \\
\hline & & & \\
\hline $\mathrm{B}$ & Bingham & & \\
\hline G & flow resistance for $\mathrm{HB}$ fluids [Nm] & $\omega$ & angular velocity $[\mathrm{rad} / \mathrm{s}](\omega=\mathrm{v} / \mathrm{r})$ \\
\hline$\hat{\mathrm{G}}$ & flow resistance for B fluids [Nm] & & \\
\hline $\mathrm{H}$ & viscosity factor for $\mathrm{HB}$ fluids $[\mathrm{Nm} \cdot \mathrm{s}]$ & \multicolumn{2}{|c|}{ Tensors } \\
\hline$\hat{\mathrm{H}}$ & viscosity factor for B fluids $[\mathrm{Nm} \cdot \mathrm{s}]$ & $2 \mathbf{D}$ & deformation rate tensor $(\equiv \dot{\gamma})($ eq. 4$)$ \\
\hline HB & Herschel-Bulkley & $\mathbf{I}$ & identity tensor (eq. 3) \\
\hline $\mathrm{h}$ & height of the inner cylinder [m] & $\widetilde{\mathbf{T}}$ & total stress tensor (eq. 3) \\
\hline $\mathrm{J}$ & flow index factor for HB fluids [-] & $\tau$ & (extra) stress tensor (eq. 3) \\
\hline $\mathrm{K}$ & consistency coefficient $\left[\mathrm{Pa} \cdot \mathrm{s}^{\mathrm{n}}\right]$ (eq. 2 ) & & \\
\hline $\mathrm{n}$ & flow index [-] (eq. 2) & \multicolumn{2}{|c|}{ Vectors } \\
\hline \multirow[t]{2}{*}{$\mathrm{N}$} & angular velocity of the outer cylinder & $\overrightarrow{\mathrm{g}}$ & gravity constant vector \\
\hline & {$[\mathrm{rps}]$} & $\overrightarrow{\mathrm{i}}_{\mathrm{x}}$ & unit vector in direction $\mathrm{x}(\mathrm{x}=\mathrm{r}, \theta, \mathrm{z})$ \\
\hline $\mathrm{p}$ & pressure $[\mathrm{Pa}]$ & $\overrightarrow{\mathrm{v}}_{\mathrm{x}}$ & velocity vector in direction $\mathrm{x}$ \\
\hline $\mathrm{r}$ & radial cylindrical coordinate $[\mathrm{m}]$ & $\overrightarrow{\mathrm{T}}$ & torque vector (eq. 24) \\
\hline $\mathrm{R}_{\mathrm{i}}$ & radius of the inner cylinder $[100 \mathrm{~mm}]$ & $\nabla$ & gradient vector (eq. 4) \\
\hline & radius of the outer cylinder $[145 \mathrm{~mm}]$ & & \\
\hline \multirow[t]{2}{*}{$\mathrm{T}$} & torque measured on inner cylinder & \multicolumn{2}{|c|}{ Functions } \\
\hline & {$[\mathrm{Nm}]$} & $\hat{\phi}$ & Lerch classical transcendent phi \\
\hline \multirow[t]{2}{*}{$\mathrm{z}$} & height cylindrical coordinate $[\mathrm{m}]$ & & function (eq. 39) \\
\hline & & $I_{\mathrm{A}}$ & $1^{\text {st }}$ invariant of tensor $\mathbf{A}(=\operatorname{tr} \mathbf{A})$ \\
\hline \multicolumn{2}{|c|}{ Greek characters } & $I I_{\mathbf{A}}$ & $2^{\text {nd }}$ invariant of tensor $\mathbf{A}$ \\
\hline & shear rate $\left[\mathrm{s}^{-1}\right]$ & & $\left(=1 / 2 \cdot\left[(\operatorname{tr} \mathbf{A})^{2}-\operatorname{tr}\left(\mathbf{A}^{2}\right)\right]\right)$ \\
\hline$\mu$ & $\begin{array}{l}\text { (dynamic) plastic viscosity for B } \\
\text { fluids }[\mathrm{Pa} \cdot \mathrm{s}] \text { (eq. } 1 \text { ) }\end{array}$ & $I I I_{\mathbf{A}}$ & $3^{\text {rd }}$ invariant of tensor $\mathbf{A}(=\operatorname{det} \mathbf{A})$ \\
\hline$\theta$ & angular cylindrical coordinate [rad] & Other & \\
\hline$\rho$ & density of test material $\left[\mathrm{kg} / \mathrm{m}^{3}\right]$ & $\mathbb{N}$ & set of natural numbers \\
\hline$\tau$ & shear stress $[\mathrm{Pa}]$ & $\mathbb{R}$ & set of real numbers \\
\hline$\tau_{0}$ & yield value for $\mathrm{HB}$ fluids $[\mathrm{Pa}]$ (eq. 1) & $\infty$ & infinity \\
\hline
\end{tabular}

\section{INTRODUCTION}

In concrete science, the rheological behaviour of the fresh concrete mix is commonly described by a Bingham (B) model. This model seems also to be valid for a whole range of self-compacting concrete (SCC) mixes. For Belgian SCC mixes, based on the powder-type philosophy of SCC mix design, a shear thickening or so-called Herschel-Bulkley (HB) behaviour of the fresh SCC mixes is quite often observed. The effect is more pronounced for mixes with higher superplasticizer contents, resulting in a very low yield value $\hat{\tau}_{0}(<10 \mathrm{~Pa})$. 
In the Herschel-Bulkley model, the shear stress $\tau$ is no longer linear proportional to the shear rate $\dot{\gamma}$ : the relation $\tau(\dot{\gamma})$ is described by a power law.

A longstanding problem in rheometry is the so-called "Couette inverse problem", in which one tries to derive the flow curve $\tau(\dot{\gamma})$ from the torque measurements $\mathrm{T}(\mathrm{N})$ in a (wide-gap) coaxial cylinder (Couette) rheometer, where $\mathrm{T}$ represents the torque registered at the inner, stationary, cylinder and $\mathrm{N}$ is the rotational velocity of the outer, rotating, cylinder. In order to solve the Couette inverse problem, 3 methods can be used: (a) the integration method: the type of constitutive equation is specified in advance and integrated to obtain the relation $T(N)$, which is fitted to the experimental data, (b) the Tikhonov regularization method, proposed by Yeow et al. [1] and (c) the wavelette-vaguelette decomposition method, proposed by Ancey [2].

In this paper, the integration method is studied in order to find out if an analytical equation can be found for converting $\mathrm{T}(\mathrm{N})$ in $\tau(\dot{\gamma})$. For a Bingham fluid, this conversion equation is known as the "Reiner-Riwlin" equation. [3] For a Herschel-Bulkley fluid, the governing conversion equation has not yet been reported. Methods (b) and (c) have not been analysed in this paper.

The rheology of fresh concrete or other cement-based material can usually, at least as a first approximation, be described by the Bingham model. In this model, the shear stress $\tau$ is assumed to be linearly proportional to the shear rate $\dot{\gamma}$, after exceeding a certain yield value $\hat{\tau}_{0}:$

$\tau= \begin{cases}0 & \tau<\hat{\tau}_{0} \\ \hat{\tau}_{0}+\mu \cdot \dot{\gamma} & \tau \geq \hat{\tau}_{0}\end{cases}$

Although there has been considerable dispute whether or not a yield value exists, it certainly is an engineering reality and there are fluids in which yielding effectively occurs. [4] Below the yield value, the fluid behaves Hookean, while it behaves Newtonian above. $\mu$ is called the plastic viscosity [Pa.s].

For a considerable number of Belgian SCC mixes, the Bingham model gives negative results for the yield value [5], which of course has no physical meaning (Figure 1). The effect seems to be even more pronounced when higher superplasticizer contents are added to the SCC mix, resulting in a lower yield value.

In order to avoid the appearance of a negative yield value, the Herschel-Bulkley model can be used to describe this material's flow behaviour. In the last model, the relation $\tau(\dot{\gamma})$, after exceeding the yield value $\tau_{0}$, is described by a power law function:

$\tau= \begin{cases}0 & \tau<\tau_{0} \\ \tau_{0}+K \cdot \dot{\gamma}^{\mathrm{n}} & \tau \geq \tau_{0}\end{cases}$

Below the yield value, the fluid behaves Hookean, while it was found that the fluid (i.e. the self-compacting concrete mix) behaves dilatant (or shear thickening: $n>1$ ) above the yield value. $\mathrm{K}$ is called the "consistency coefficient" $[\mathrm{Pa} \cdot \mathrm{s}$ "], where $\mathrm{n}$ denotes the "flow index" [-]. 


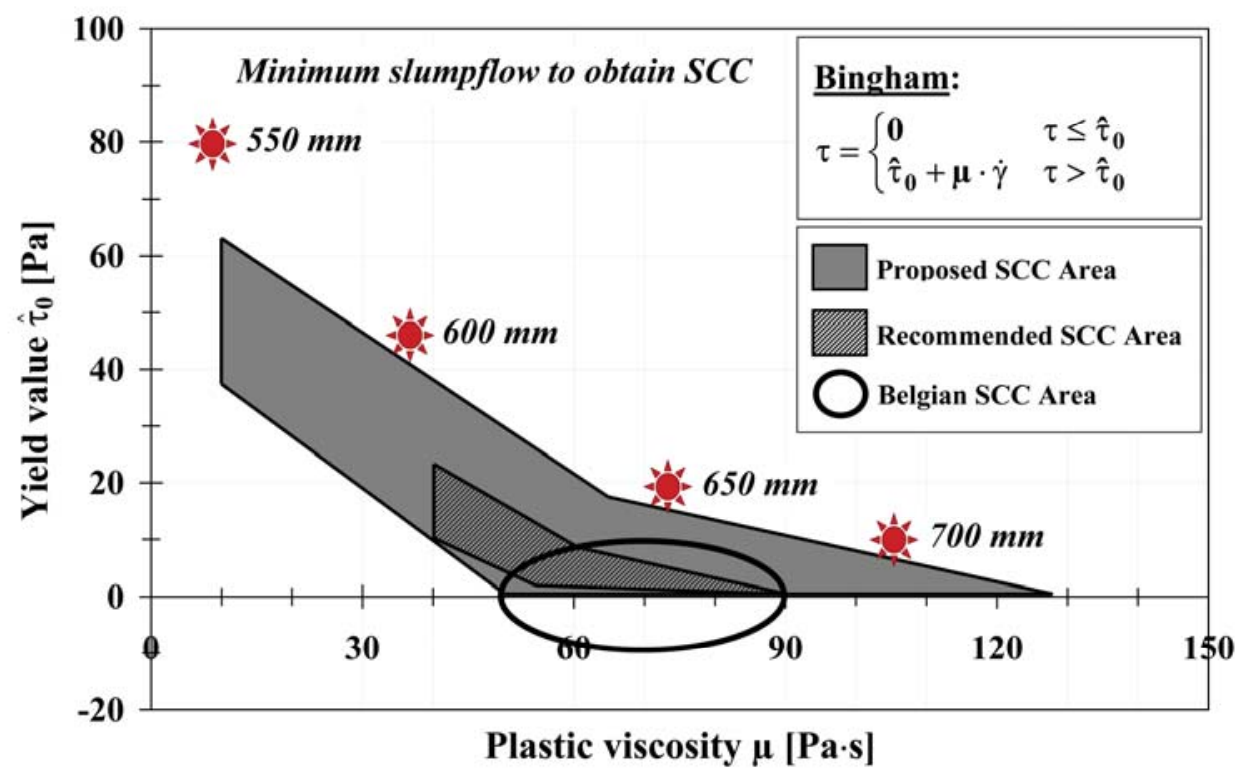

Figure 1: Defining SCC in terms of its Bingham characteristics (after Wallevik, Ó. [6])

\section{THE COUETTE RHEOMETER \& CONCRETE SCIENCE}

A schematic 2D top view of the Couette rheometer under consideration is shown in Figure 2. An example of such apparatus would be the "ConTec Visco5", shown in Figure 3 and 4.

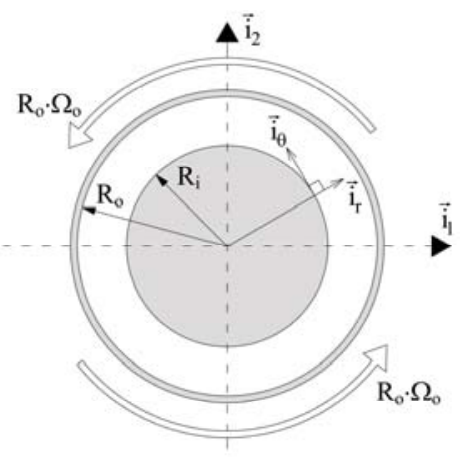

Figure 2: Schematic top view

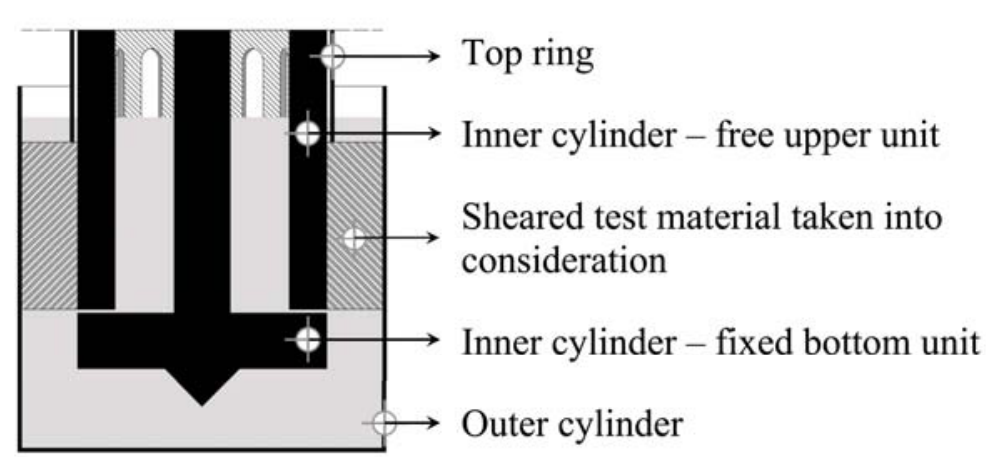

Figure 3: Schematic cross-section of the ConTec Visco5

of the Couette rheometer

The ConTec Visco5 is one of the most recent updates of the BML viscometer [7], well designed for testing both (self-compacting) concrete and mortar $\left(\mathrm{D}_{\max } \leq 22 \mathrm{~mm}\right.$ ). The outer cylinder (radius $\mathrm{R}_{\mathrm{o}}$ ) rotates at angular velocity $\Omega_{\mathrm{o}} \equiv 2 \pi \mathrm{N}[\mathrm{rad} / \mathrm{s}]$, while the inner cylinder (radius $\mathrm{R}_{\mathrm{i}}$ ) is stationary and registers the applied torque $\mathrm{T}$ [Nm] from the rheological continuum (i.e. in our case, the self-compacting concrete).

The measuring system consists of an outer cylinder $\left(\mathrm{R}_{\mathrm{o}}=145 \mathrm{~mm}\right)$, an inner cylinder unit (standard unit C-200: $\mathrm{R}_{\mathrm{i}}=100 \mathrm{~mm}$ ) and a top ring. The outer cylinder is well mounted on a rotating disk. The inner cylinder is lowered into the outer cylinder by a screw-jack system. 
The inner cylinder is constructed as a two component unit: a bottom unit, which is fixed at the mounting point of the inner cylinder, and a upper unit, which is free to rotate against a load cell, registering the applied torque $\mathrm{T}$ from the rheological continuum. Both inner and outer cylinder are equipped with steel ribs in order to eliminate/reduce slip at their surfaces.

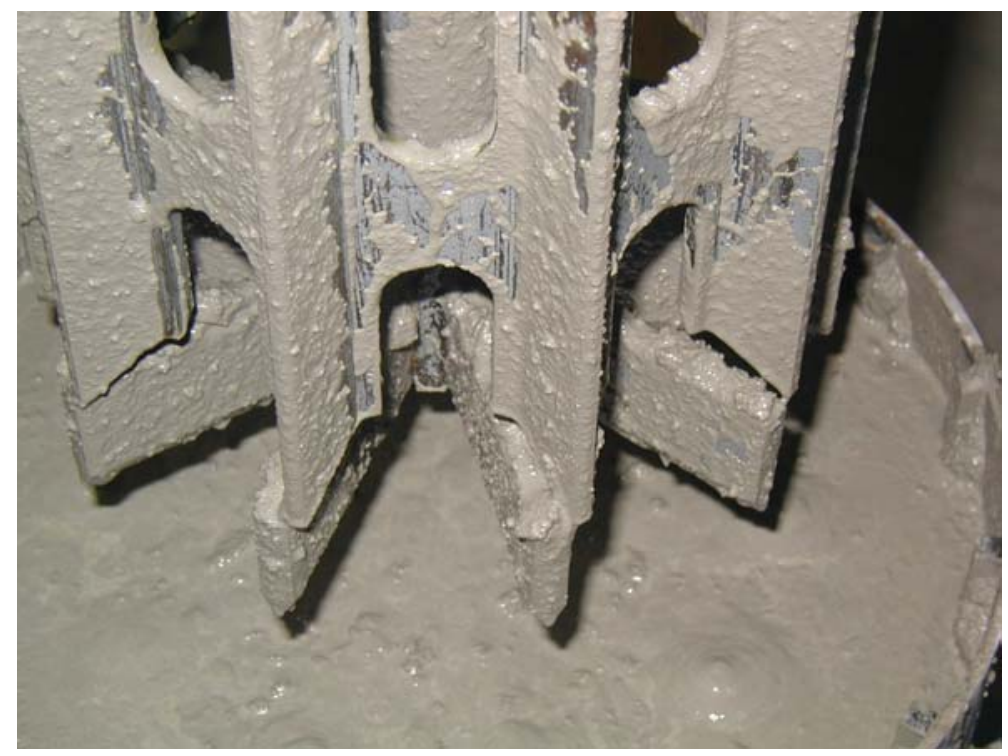

Figure 4: Detailed view of inner cylinder and bottom unit of the ConTec Visco5

The arrangement of the two component inner cylinder will virtually eliminate the effect of $3 \mathrm{D}$ shearing at the bottom of the inner cylinder and therefore requires no special correction regarding possible bottom effects. The top ring is fitted over the inner cylinder, in order to insure a constant height of the sheared test material.

\section{THE CONSTITUTIVE EQUATION}

In order to describe the state of stress at any point in the material, the total stress tensor $\widetilde{\mathbf{T}}$ is used. [8] It linearly transforms the normal vector of a plane, through a given point, into the corresponding stress vector acting on that plane:

$\widetilde{\mathbf{T}}=-\mathrm{p} \cdot \mathbf{I}+\tau$

with $\widetilde{\mathbf{T}}$ the total stress tensor, $\mathrm{p}$ the isotropic pressure, $\mathbf{I}$ the identity tensor $\left(\mathrm{I}_{\mathrm{ij}} \equiv \delta_{\mathrm{ij}}\right)$ and $\tau$ the (extra) stress tensor. In the case an incompressible material is assumed, it will be the stress tensor $\tau$ which reflects the material's deformation and therefore the one to be used in constitutive equations.

In order to describe the deformation (or strain) rate at any point in the material, the deformation rate tensor 2D ( $\equiv \dot{\gamma}$ for simple shear) is used. [8] It operates on small displacement vectors around a point in the material to give the time rate of change of the squared length of those vectors:

$$
2 \mathbf{D}=\mathbf{L}+\mathbf{L}^{\mathrm{T}}=(\nabla \overrightarrow{\mathbf{v}})^{\mathrm{T}}+(\nabla \overrightarrow{\mathbf{v}})
$$


with $\mathbf{L}$ the velocity gradient tensor and $\nabla \overrightarrow{\mathbf{v}}$ the dyad product of the gradient vector $\nabla$ and the velocity vector $\overrightarrow{\mathrm{v}}$.

For a general viscous fluid, it is assumed that stress depends only on the deformation rate [8], or better, on an invariant of the deformation rate tensor, as it is shown further on. To enable describing non-linear material (flow) properties, the governing equation has to be made non-linear:

$$
\widetilde{\mathbf{T}}=\mathrm{f}(2 \mathbf{D}) \equiv \mathrm{a}_{0} \cdot(2 \mathbf{D})^{0}+\mathrm{a}_{1} \cdot(2 \mathbf{D})^{1}+\mathrm{a}_{2} \cdot(2 \mathbf{D})^{2}+\mathrm{a}_{3} \cdot(2 \mathbf{D})^{3}+\ldots
$$

where $(2 \mathbf{D})^{0} \equiv \mathbf{I}$ and, for an incompressible fluid, $\mathrm{a}_{0} \equiv-$ p. Evoking the Cayley-Hamilton theorem, which quotes that all terms of higher order then 2 will be zero as we are working in the $3 \mathrm{D}$ space, equation (5) can be written as:

$\widetilde{\mathbf{T}}=-\mathrm{p} \cdot \mathbf{I}+\eta_{1}\left(I_{2 \mathbf{D}}, I I_{2 \mathbf{D}}, I I_{2 \mathbf{D}}\right) \cdot 2 \mathbf{D}+\eta_{2}\left(I_{2 \mathbf{D}}, I I_{2} \mathbf{D}, I I_{2 \mathbf{D}}\right) \cdot(2 \mathbf{D})^{2}$

where $\eta_{1}$ and $\eta_{2}$ are scalar functions of the invariants of $2 \mathbf{D}$. This equation is known as the "Reiner-Rivlin" constitutive equation for general viscous fluids.

Furthermore, still assuming an incompressible fluid, $I_{2 \mathbf{D}}=\operatorname{tr}(2 \mathbf{D})=\nabla \cdot \overrightarrow{\mathrm{v}} \equiv 0$ and, because of the symmetry of $2 \mathbf{D}$ (which is the case for most fluids), $I I I_{2 \mathbf{D}}=\operatorname{det}(2 \mathbf{D}) \equiv 0$. Besides, since the $\eta_{2}$ term gives qualitatively the wrong result, it is usually discarded. [8]

Therefore, equation (6) reduces to:

$\widetilde{\mathbf{T}}=-\mathrm{p} \cdot \mathbf{I}+\eta_{1}\left(I I_{2 \mathbf{D}}\right) \cdot 2 \mathbf{D}$

When comparing equations (3) and (7), the following relation is found $\left(\eta_{1} \equiv \eta\right)$ :

$\tau=\eta\left(I I_{2 \mathbf{D}}\right) \cdot 2 \mathbf{D}$

By this last equation, equations (1) and (2) can be generalized, i.e. put into a 3D form, for deformations occurring in more than one direction. The only significant change required, is to replace the $1 \mathrm{D}$ yield criterion with some scalar function of the invariants of $\tau$. The von Mises criterion, using the second invariant of $\tau$, is most commonly used. For a Bingham and Herschel-Bulkley fluid respectively, the following constitutive equations are found for the stress tensor, after exceeding the yield value:

$$
\begin{array}{ll}
\boldsymbol{\tau}=\left(\frac{\hat{\tau}_{0}}{\left|I I_{2 \mathbf{D}}\right|^{1 / 2}}+\mu\right) \cdot 2 \mathbf{D} & \text { for } I I_{\boldsymbol{\tau}} \geq \hat{\tau}_{0} \\
\boldsymbol{\tau}=\left(\frac{\tau_{0}}{\left|I I_{2 \mathbf{D}}\right|^{1 / 2}}+\mathrm{K} \cdot\left|I I_{2 \mathbf{D}}\right|^{(\mathrm{n}-1) / 2}\right) \cdot 2 \mathbf{D} & \text { for } I I_{\boldsymbol{\tau}} \geq \tau_{0}
\end{array}
$$

\section{SHEAR STRESS \& SHEAR RATE IN A WIDE-GAP COUETTE RHEOMETER}

The deformation of a material is not only governed by the previously mentioned constitutive equation between deformation and stress, it must also obey the principles of conservation of both mass and momentum (as part of the Navier-Stokes equations): 
Mass balance: $\frac{\Delta \rho}{\Delta \mathrm{t}}=-\rho \nabla \cdot \overrightarrow{\mathrm{v}} \Rightarrow \nabla \cdot \overrightarrow{\mathrm{v}}=0$ for an incompressible material $(\rho=\underline{\mathrm{cst}})$

Momentum balance: $\rho \frac{\Delta \overrightarrow{\mathrm{v}}}{\Delta \mathrm{t}}=\nabla \cdot \widetilde{\mathbf{T}}+\rho \overrightarrow{\mathrm{g}}$

where $\rho$ is the density of the test material and $\vec{g}$ is the gravity constant vector.

Detailed derivations of these equations are available in literature on fluid mechanics. In fact, it will be the mass balance and the momentum balance which will be used to derive the general formulas for the shear stress and shear rate in a wide-gap Couette rheometer, as shown below.

\subsection{Velocity}

By using the general velocity $\vec{v}=v_{r}(r, \theta, z, t) \vec{i}_{r}+v_{\theta}(r, \theta, z, t) \vec{i}_{\theta}+v_{z}(r, \theta, z, t) \vec{i}_{z}$, it is only possible to obtain a solution for the inverse Couette problem by numerical means. However, some reasonable assumptions about the flow can be made, [9] which make an analytical approach possible:

- With low Reynolds number (i.e. sufficiently low shear rate and sufficiently high shear viscosity) the flow is assumed to be (a) stable, i.e. laminar, no secondary flows and (b) symmetrical in z-direction:

$\vec{v}=v_{r}(r, \theta, z, t) \vec{i}_{r}+v_{\theta}(r, \theta, z, t) \vec{i}_{\theta}$

- Since bottom and top effects are eliminated by geometrical means (fixed bottom unit of inner cylinder and top ring, see above), the velocity profile is height independent:

$$
\overrightarrow{\mathrm{v}}=\mathrm{v}_{\mathrm{r}}(\mathrm{r}, \theta, \mathrm{t}) \overrightarrow{\mathrm{i}}_{\mathrm{r}}+\mathrm{v}_{\theta}(\mathrm{r}, \theta, \mathrm{t}) \overrightarrow{\mathrm{i}}_{\theta}
$$

- Due to the circular geometry of the Couette rheometer, the flow is assumed to be purely circular with angle independence:

$\overrightarrow{\mathrm{v}}=\mathrm{v}_{\theta}(\mathrm{r}, \mathrm{t}) \overrightarrow{\mathrm{i}}_{\theta}$

- Since each measurement is applied in equilibrium conditions (equilibrium shear stress at each shear rate) the flow is assumed to be steady state, i.e. time independent for the given measurement:

$$
\overrightarrow{\mathrm{v}}=\mathrm{v}_{\theta}(\mathrm{r}) \overrightarrow{\mathrm{i}}_{\theta}
$$

At the moment, still little is known about the velocity profile itself, but the three following observations can be made:

- The velocity at the inner cylinder is zero because of the "no slip" condition.

- The velocity at the outer cylinder is $\mathrm{v}_{\theta}\left(\mathrm{R}_{\mathrm{o}}\right) \equiv \mathrm{R}_{\mathrm{o}} \Omega_{\mathrm{o}}$, due to the same reason as above.

- Equation (12) is generally parabolic in nature, due to it's viscous term. [10] So, from the above two points, the velocity increases monotonically from the rheometer inner cylinder $\left(\mathrm{R}_{\mathrm{i}}\right)$ to its outer cylinder $\left(\mathrm{R}_{\mathrm{o}}\right)$ with decreasing slope: 


$$
\frac{\partial \mathrm{v}_{\theta}(\mathrm{r})}{\partial \mathrm{r}} \geq 0 \quad \wedge \quad \frac{\partial^{2} \mathrm{v}_{\theta}(\mathrm{r})}{\partial \mathrm{r}^{2}} \leq 0 \quad \forall \mathrm{r} \in\left[\mathrm{R}_{\mathrm{i}}, \mathrm{R}_{\mathrm{o}}\right]
$$

\subsection{Shear rate}

Introducing equation (16) into equation (4), the rate of deformation (or shear rate) can easily be determined. Because the velocity gradient tensor $\mathbf{L}$ is given by:

$$
\mathbf{L}=\left[\begin{array}{ccc}
\mathrm{L}_{\mathrm{rr}} & \mathrm{L}_{\mathrm{r} \theta} & \mathrm{L}_{\mathrm{rz}} \\
\mathrm{L}_{\theta \mathrm{r}} & \mathrm{L}_{\theta \theta} & \mathrm{L}_{\theta \mathrm{z}} \\
\mathrm{L}_{\mathrm{zr}} & \mathrm{L}_{\mathrm{z} \theta} & \mathrm{L}_{\mathrm{zz}}
\end{array}\right]=\left[\begin{array}{cccc}
\frac{\partial \mathrm{v}_{\mathrm{r}}}{\partial \mathrm{r}} & \frac{1}{\mathrm{r}} \frac{\partial \mathrm{v}_{\mathrm{r}}}{\partial \theta}-\frac{\mathrm{v}_{\theta}}{\mathrm{r}} & \frac{\partial \mathrm{v}_{\mathrm{r}}}{\partial \mathrm{z}} \\
\frac{\partial \mathrm{v}_{\theta}}{\partial \mathrm{r}} & \frac{1}{\mathrm{r}} \frac{\partial \mathrm{v}_{\theta}}{\partial \theta}+\frac{\mathrm{v}_{\mathrm{r}}}{\mathrm{r}} & \frac{\partial \mathrm{v}_{\theta}}{\partial \mathrm{z}} \\
\frac{\partial \mathrm{v}_{\mathrm{z}}}{\partial \mathrm{r}} & \frac{1}{\mathrm{r}} \frac{\partial \mathrm{v}_{\mathrm{z}}}{\partial \theta} & \frac{\partial \mathrm{v}_{\mathrm{z}}}{\partial \mathrm{z}}
\end{array}\right]
$$

the deformation rate tensor $2 \mathbf{D}$ can be calculated as:

$$
2 \mathbf{D}=\mathbf{L}+\mathbf{L}^{\mathrm{T}}=\left[\begin{array}{ccc}
0 & -\frac{\mathrm{v}_{\theta}}{\mathrm{r}} & 0 \\
\frac{\partial \mathrm{v}_{\theta}}{\partial \mathrm{r}} & 0 & 0 \\
0 & 0 & 0
\end{array}\right]+\left[\begin{array}{ccc}
0 & \frac{\partial \mathrm{v}_{\theta}}{\partial \mathrm{r}} & 0 \\
-\frac{\mathrm{v}_{\theta}}{\mathrm{r}} & 0 & 0 \\
0 & 0 & 0
\end{array}\right]=\left[\begin{array}{ccc}
0 & \frac{\partial \mathrm{v}_{\theta}}{\partial \mathrm{r}}-\frac{\mathrm{v}_{\theta}}{\mathrm{r}} & 0 \\
\frac{\partial \mathrm{v}_{\theta}}{\partial \mathrm{r}}-\frac{\mathrm{v}_{\theta}}{\mathrm{r}} & 0 & 0 \\
0 & 0 & 0
\end{array}\right]
$$

or:

$\dot{\gamma}=\frac{\partial \mathrm{v}_{\theta}(\mathrm{r})}{\partial \mathrm{r}}-\frac{\mathrm{v}_{\theta}(\mathrm{r})}{\mathrm{r}} \equiv \mathrm{r} \frac{\partial \omega(\mathrm{r})}{\partial \mathrm{r}}$

with $\omega(r)$ the angular velocity at radius $r$.

As an illustration, Figure 5 shows a plot of both the velocity and shear rate profile in the ConTec Visco5 for a Bingham fluid with the following properties: $\mu=63 \mathrm{~Pa} \cdot \mathrm{s}, \hat{\tau}_{0}=18 \mathrm{~Pa}$, $\mathrm{N}=0.25 \mathrm{rps}, \mathrm{R}_{\mathrm{i}}=0.100 \mathrm{~m}, \mathrm{R}_{\mathrm{o}}=0.145 \mathrm{~m}$ and $\mathrm{h}=0.130 \mathrm{~m}$.

It may be clear from this figure that no constant shear rate can be assumed when making theoretical calculations based on wide-gap rheometry: for this particular example, the shear rate increases by more than $100 \%$ from the outer cylinder to the inner one.

Besides, it is worth notice that the shear rate, contrary to the shear stress (see further), depends on both the rheometer geometry and the nature of the fluid (at steady state). 

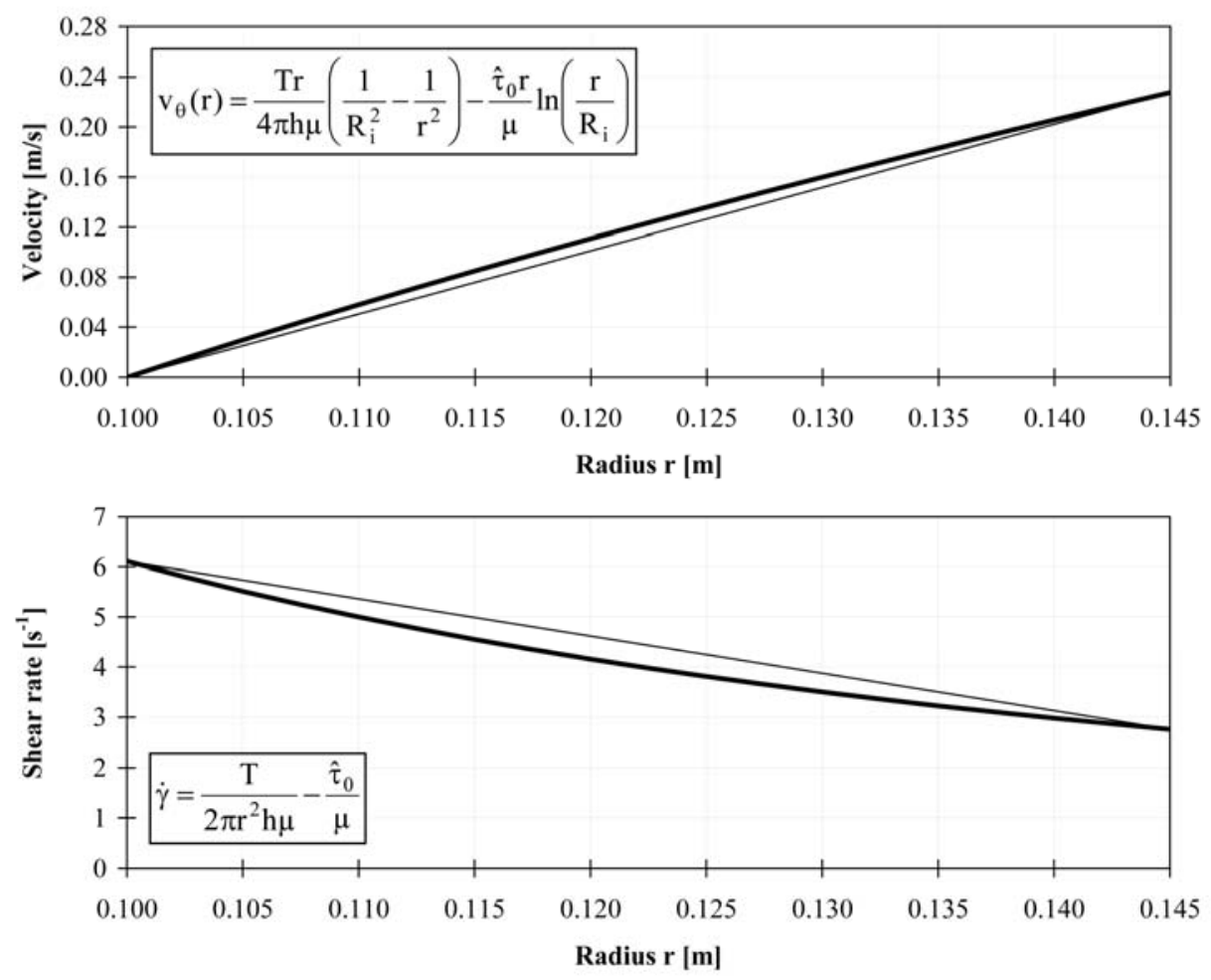

Figure 5: Example of the velocity and shear rate profile for a Bingham fluid in a wide-gap Couette rheometer, eq. (20) and (33)

\subsection{Shear stress}

In general, the decomposition of the conservation of momentum (eq. (12)) in $\theta$-direction, using equation (3) to express the result in function of the stress tensor $\tau$, is given by:

$$
\begin{aligned}
& \rho\left(\frac{\partial \mathrm{v}_{\theta}}{\partial \mathrm{t}}+\mathrm{v}_{\mathrm{r}} \frac{\partial \mathrm{v}_{\theta}}{\partial \mathrm{r}}+\frac{\mathrm{v}_{\theta}}{\mathrm{r}} \frac{\partial \mathrm{v}_{\theta}}{\partial \theta}+\frac{\mathrm{v}_{\mathrm{r}} \mathrm{v}_{\theta}}{\mathrm{r}}+\mathrm{v}_{\mathrm{z}} \frac{\partial \mathrm{v}_{\theta}}{\partial \mathrm{z}}\right)= \\
& -\frac{1}{\mathrm{r}} \frac{\partial \mathrm{p}}{\partial \theta}+\left(\frac{1}{\mathrm{r}^{2}} \frac{\partial\left(\mathrm{r}^{2} \tau_{\mathrm{r} \theta}\right)}{\partial \mathrm{r}}+\frac{1}{\mathrm{r}} \frac{\partial \tau_{\theta \theta}}{\partial \theta}+\frac{\partial \tau_{\theta \mathrm{z}}}{\partial \mathrm{z}}+\frac{\tau_{\theta \mathrm{r}}-\tau_{\mathrm{r} \theta}}{\mathrm{r}}\right)+\rho \mathrm{g}_{\theta}
\end{aligned}
$$

which can be strongly simplified by using equation (16) of the velocity vector, resulting in:

$$
\frac{1}{\mathrm{r}^{2}} \frac{\partial\left(\mathrm{r}^{2} \tau_{\mathrm{r} \theta}\right)}{\partial \mathrm{r}}=0 \text { or } \frac{\partial\left(\tau_{\mathrm{r} \theta}\right)}{\partial \mathrm{r}}+\frac{2}{\mathrm{r}} \tau_{\mathrm{r} \theta}=0
$$

A particular solution of this last, first-order homogeneous linear differential equation is found by integration from the inner radius $R_{i}$ to an arbitrary radius $r \in\left[R_{i}, R_{o}\right]$, imposing the boundary condition $\tau_{\mathrm{r} \theta}(\mathrm{r}) \equiv \tau_{\mathrm{r} \theta}\left(\mathrm{R}_{\mathrm{i}}\right)$ for $\mathrm{r}=\mathrm{R}_{\mathrm{i}}$ :

$\tau_{\mathrm{r} \theta}(\mathrm{r})=\tau_{\mathrm{r} \theta}\left(\mathrm{R}_{\mathrm{i}}\right)\left(\frac{\mathrm{R}_{\mathrm{i}}}{\mathrm{r}}\right)^{2}$ 
In general, the torque $\vec{T}$ applied on a cylindrical shell from its outer material is calculated with the help of Cauchy's stress principle [11], expressing equivalence between applied torque and shear stresses on the shell:

$\overrightarrow{\mathrm{T}}=\mathrm{T} \overrightarrow{\mathrm{i}}_{\mathrm{z}}=\int_{0}^{\mathrm{h}} \int_{0}^{2 \pi} \mathrm{r} \overrightarrow{\mathrm{i}}_{\mathrm{r}} \times\left(\overrightarrow{\mathrm{i}}_{\mathrm{r}} \cdot \boldsymbol{\tau}(\mathrm{r}) \mathrm{rd} \theta \mathrm{dz}\right)=2 \pi \mathrm{r}^{2} \mathrm{~h} \tau_{\mathrm{r} \theta}(\mathrm{r}) \overrightarrow{\mathrm{i}}_{\mathrm{z}}$

with $\mathrm{h}$ the height of the considered cylindrical shell. In the same way, the torque applied from the test material on the inner cylinder ( $\mathrm{r} \equiv \mathrm{R}_{\mathrm{i}}$ in the above equation) can be determined, resulting in (by also using equation (23)):

$\mathrm{T}=2 \pi \mathrm{R}_{\mathrm{i}}^{2} \mathrm{~h} \tau_{\mathrm{r} \theta}\left(\mathrm{R}_{\mathrm{i}}\right)$ or $\tau_{\mathrm{r} \theta}(\mathrm{r})=\frac{\mathrm{T}}{2 \pi \mathrm{r}^{2} \mathrm{~h}}$

The latter equation shows that the shear stress of a fluid in a Couette rheometer depends only on the rheometer geometry and not on the nature of the fluid (at steady state).

\section{SOLVING THE COUETTE INVERSE PROBLEM FOR A BINGHAM FLUID BY THE INTEGRATION METHOD: THE "REINER-RIWLIN" EQUATION}

Equation (1) can be rewritten, using equations (20) and (25), as:

$\frac{\mathrm{T}}{2 \pi \mathrm{r}^{2} \mathrm{~h}}=\hat{\tau}_{0}+\mu \cdot \mathrm{r} \frac{\partial \omega(\mathrm{r})}{\partial \mathrm{r}}$ for $\tau \geq \hat{\tau}_{0}$

Assuming "no slip" boundary conditions, i.e. $r=R_{i} \rightarrow \omega \equiv 0 \wedge r=R_{0} \rightarrow \omega \equiv \Omega_{0}$, the above equation can be integrated as follows:

$\int_{\mathrm{R}_{\mathrm{i}}}^{\mathrm{R}_{\mathrm{o}}}\left(\frac{\mathrm{T}}{2 \pi \mathrm{r}^{3} \mathrm{~h}}-\frac{\hat{\tau}_{0}}{\mathrm{r}}\right) \mathrm{dr}=\mu \int_{0}^{\Omega_{\mathrm{o}}} \mathrm{d} \omega(\mathrm{r})$

Which can be solved analytically (using $\Omega_{\mathrm{o}} \equiv 2 \pi \mathrm{N}$ ):

$\frac{\mathrm{T}}{2 \pi \mathrm{h}} \int_{\mathrm{R}_{\mathrm{i}}}^{\mathrm{R}_{\mathrm{o}}} \frac{\mathrm{dr}}{\mathrm{r}^{3}}-\hat{\tau}_{0} \int_{\mathrm{R}_{\mathrm{i}}}^{\mathrm{R}_{\mathrm{o}}} \frac{\mathrm{dr}}{\mathrm{r}}=\mu \Omega_{\mathrm{o}}$

and thus:

$\frac{\mathrm{T}}{4 \pi \mathrm{h}}\left(\frac{1}{\mathrm{R}_{\mathrm{i}}^{2}}-\frac{1}{\mathrm{R}_{\mathrm{o}}^{2}}\right)-\hat{\tau}_{0} \ln \left(\frac{\mathrm{R}_{\mathrm{o}}}{\mathrm{R}_{\mathrm{i}}}\right)=\mu(2 \pi \mathrm{N})$

Rearranging the latter equation for $\mathrm{T}$, the well-known "Reiner-Riwlin" equation is found:

$\mathrm{T}=\frac{4 \pi \mathrm{h} \hat{\tau}_{0}}{\left(\frac{1}{\mathrm{R}_{\mathrm{i}}^{2}}-\frac{1}{\mathrm{R}_{\mathrm{o}}^{2}}\right)} \ln \left(\frac{\mathrm{R}_{\mathrm{o}}}{\mathrm{R}_{\mathrm{i}}}\right)+\frac{\mu 8 \pi^{2} \mathrm{~h}}{\left(\frac{1}{\mathrm{R}_{\mathrm{i}}^{2}}-\frac{1}{\mathrm{R}_{\mathrm{o}}^{2}}\right)} \mathrm{N} \equiv \hat{\mathrm{G}}+\hat{\mathrm{H} N}$ 
where $\hat{\mathrm{G}}$ and $\hat{\mathrm{H}}$ are respectively the flow resistance and the viscosity factor for a Bingham fluid.

So:

$\hat{\tau}_{0}=\frac{\hat{\mathrm{G}}}{4 \pi \mathrm{h}}\left(\frac{1}{\mathrm{R}_{\mathrm{i}}^{2}}-\frac{1}{\mathrm{R}_{\mathrm{o}}^{2}}\right) \frac{1}{\ln \left(\mathrm{R}_{\mathrm{o}} / \mathrm{R}_{\mathrm{i}}\right)}$

and

$\mu=\frac{\hat{\mathrm{H}}}{8 \pi^{2} \mathrm{~h}}\left(\frac{1}{\mathrm{R}_{\mathrm{i}}^{2}}-\frac{1}{\mathrm{R}_{\mathrm{o}}^{2}}\right)$

Equation (30) is the solution of the inverse (wide-gap) Couette problem for a Bingham fluid, since the following steps can be made in order to derive the flow curve $\tau(\dot{\gamma})$ from the torque measurements $\mathrm{T}(\mathrm{N})$ :

- determine $\hat{\mathrm{G}}$ and $\hat{\mathrm{H}}$ by fitting the experimental data $\mathrm{T}(\mathrm{N})$ into $\mathrm{T}=\hat{\mathrm{G}}+\hat{\mathrm{H} N}$ (keeping in mind that a steady state flow and no appearance of slip nor plug are required).

- determine $\hat{\tau}_{0}$ and $\mu$, according to equation (31) and (32) respectively, and fill in their values into equation (1).

Note: when changing the "outer" boundary condition into $r=r \rightarrow \omega \equiv v_{\theta}(r) / r$, equation (27) can be solved for the velocity profile (see Figure 5):

$\mathrm{v}_{\theta}(\mathrm{r})=\frac{\operatorname{Tr}}{4 \pi \mathrm{h} \mu}\left(\frac{1}{\mathrm{R}_{\mathrm{i}}^{2}}-\frac{1}{\mathrm{r}^{2}}\right)-\frac{\hat{\tau}_{0} \mathrm{r}}{\mu} \ln \left(\frac{\mathrm{r}}{\mathrm{R}_{\mathrm{i}}}\right)$

\section{SOLVING THE COUETTE INVERSE PROBLEM FOR A HERSCHEL- BULKLEY FLUID BY THE INTEGRATION METHOD}

Since equation (20) and (25) are not depending on any rheological model, they can also be used in the case of a Herschel-Bulkley fluid, so equation (2) can be written as:

$$
\frac{\mathrm{T}}{2 \pi \mathrm{r}^{2} \mathrm{~h}}=\tau_{0}+\mathrm{K} \cdot\left(\mathrm{r} \frac{\partial \omega(\mathrm{r})}{\partial \mathrm{r}}\right)^{\mathrm{n}} \text { for } \tau \geq \tau_{0} .
$$

The same "no slip" boundary conditions as mentioned for the Bingham fluid can be applied, resulting in the following integration of equation (34):

$\int_{R_{i}}^{R_{0}}\left(\left(\frac{T}{2 \pi r^{2} h K}-\frac{\tau_{0}}{K}\right)^{1 / n} \frac{1}{r}\right) d r=\int_{0}^{\Omega_{0}} d \omega(r)$

or:

$\int_{\mathrm{R}_{\mathrm{i}}}^{\mathrm{R}_{\mathrm{o}}}\left(\left(\frac{\mathrm{T}}{2 \pi \mathrm{r}^{2} \mathrm{hK}}-\frac{\tau_{0}}{\mathrm{~K}}\right)^{1 / \mathrm{n}} \frac{1}{\mathrm{r}}\right) \mathrm{dr}=\Omega_{\mathrm{o}}=2 \pi \mathrm{N}$ 
First, the left side of the latter equation is rewritten as:

$\int_{\mathrm{R}_{\mathrm{i}}}^{\mathrm{R}_{\mathrm{o}}}\left(\left(\frac{\alpha \mathrm{T}}{\mathrm{r}^{2}}-\beta\right)^{1 / \mathrm{n}} \frac{1}{\mathrm{r}}\right) \mathrm{dr}$ with $\alpha=\frac{1}{2 \pi \mathrm{hK}}$ and $\beta=\frac{\tau_{0}}{\mathrm{~K}}$

To solve equation (37), the following substitution is used:

$\chi=\frac{\alpha \mathrm{T}}{\mathrm{r}^{2}}-\beta \Rightarrow \frac{\mathrm{d} \chi}{\mathrm{dr}}=-2 \frac{\alpha \mathrm{T}}{\mathrm{r}^{3}} \Rightarrow \frac{\mathrm{dr}}{\mathrm{r}}=-\frac{\mathrm{r}^{2} \mathrm{~d} \chi}{2 \alpha \mathrm{T}}=-\frac{1}{2} \frac{\mathrm{d} \chi}{\chi+\beta}$

So, equation (37) becomes:

$-\frac{1}{2} \int_{\chi_{i}}^{\chi_{0}} \frac{\chi^{1 / \mathrm{n}}}{\chi+\beta} \mathrm{d} \chi \equiv-\frac{1}{2}\left[\chi^{1 / \mathrm{n}}\left[\mathrm{n}-\hat{\phi}\left(-\frac{\chi}{\beta}, 1, \frac{1}{\mathrm{n}}\right)\right]\right]_{\chi_{\mathrm{i}}}^{\chi_{0}}$

where $\hat{\phi}$ denotes the "Lerch classical transcendent Phi" function, a single-valued, continuous (non-polynomial) function on the $\mathrm{z}$-plane cut along the interval $\mathrm{z} \in$ ] $-\infty, 1[$ (for fixed $\mathrm{a},-\mathrm{a} \notin$ $\mathbb{N})$.

An evaluation of $\hat{\phi}(z, 1, a)$ for discrete values of a $(\equiv 1 / n)$ is given in Figure 6 . Note that:

- for $\left.\mathrm{a}=1, \hat{\phi}(\mathrm{z}, 1, \mathrm{a})=\hat{\phi}(\mathrm{z}, 1,1) \equiv-\frac{\ln (1-\mathrm{z})}{\mathrm{z}} \forall \mathrm{z} \in\right]-\infty, 0[\cup] 0,1[$

- for $\mathrm{z}=0, \hat{\phi}(\mathrm{z}, 1, \mathrm{a})=\hat{\phi}(0,1, \mathrm{a}) \equiv \frac{1}{|\mathrm{a}|} \equiv|\mathrm{n}| \quad \forall \mathrm{a} \in \mathbb{R}$

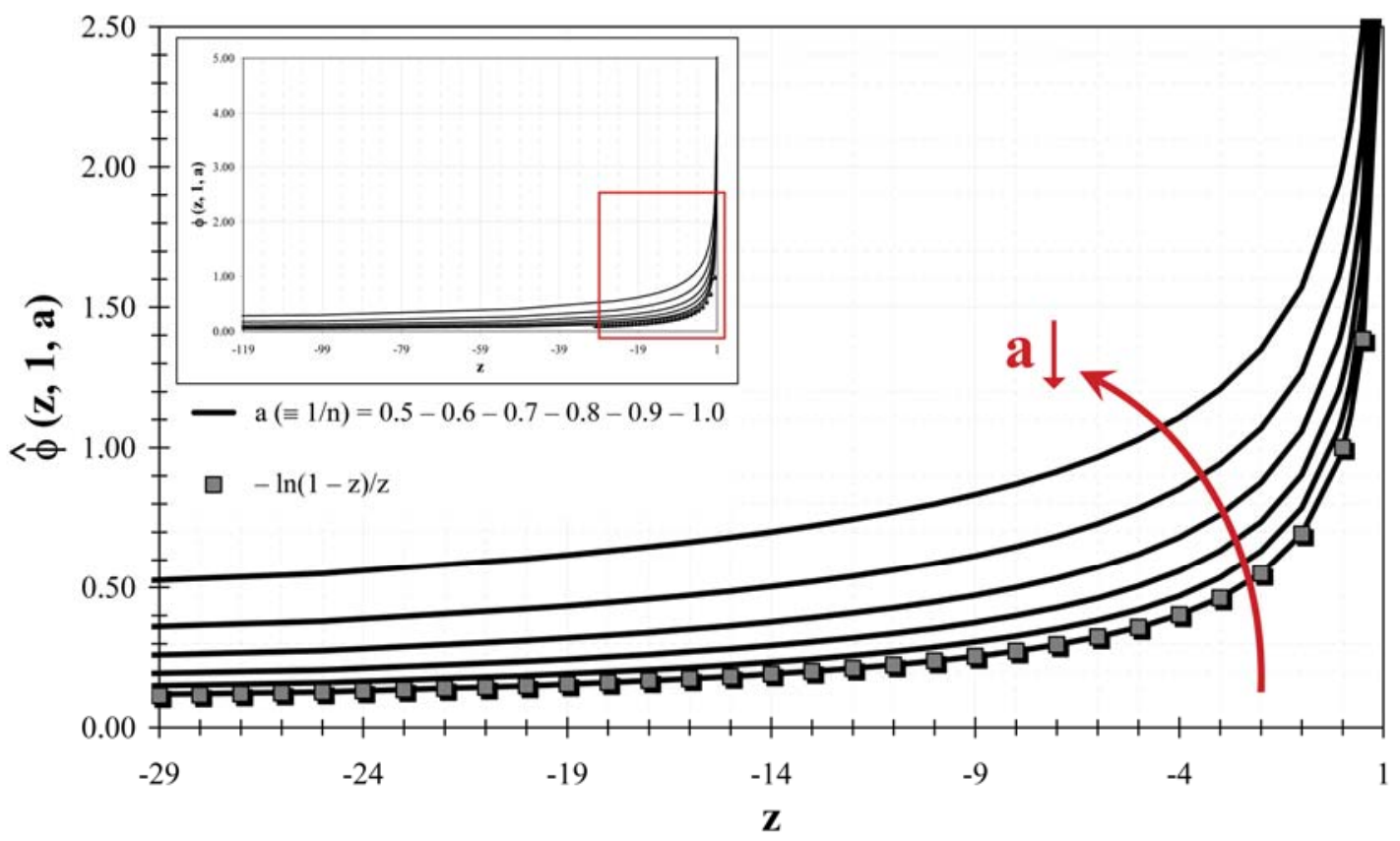

Figure 6: $\hat{\phi}(\mathrm{z}, 1, \mathrm{a})$ - Evaluation for some discrete values of $\mathrm{a}(1 \leq \mathrm{n} \leq 2)$ 
By re-substitution of equation (39), equation (36) becomes:

$$
\left[\left(\frac{\mathrm{T}}{2 \pi \mathrm{R}_{\mathrm{i}}^{2} \mathrm{hK}}-\frac{\tau_{0}}{\mathrm{~K}}\right)^{1 / \mathrm{n}}\left[\mathrm{n}-\hat{\phi}\left(1-\frac{\mathrm{T}}{2 \pi \mathrm{R}_{\mathrm{i}}^{2} \mathrm{~h} \tau_{0}}, 1, \frac{1}{\mathrm{n}}\right)\right]-\right]=4 \pi \mathrm{N}
$$

According to the Bingham fluid, the latter equation should preferably be transformed into the general form:

$$
\mathrm{T}=\mathrm{G}+\mathrm{HN}^{\mathrm{J}}
$$

with $\mathrm{G}$ the flow resistance, $\mathrm{H}$ the viscosity factor and $\mathrm{J}$ the flow index factor for a HerschelBulkley fluid.

According to [8], the flow index $\mathrm{n}$ as defined in equation (2) is equal to the flow index factor J, defined by equation (43):

$\mathrm{n} \equiv \mathrm{J}=\frac{\partial \ln (\mathrm{T}-\mathrm{G})}{\partial \ln \mathrm{N}}$

Note that in [8] the equality $\mathbf{J} \equiv \mathrm{n}$ is given for a power-law fluid $\left(\tau=\mathrm{K} \cdot \dot{\gamma}^{\mathrm{n}}\right)$, without any restriction related to the gap width. However, it is not unreasonable to assume that the same equality is also valid for a Herschel-Bulkley fluid, after exceeding the yield value.

\subsection{Solution for a Bingham fluid $(\mathrm{n}=1)$}

Before evaluating equation (42) for $n \neq 1$, the applicability of this equation for $n=1$ will be established. For this purpose, equation (40) will be used to rewrite equation (42) as follows:

$$
\left(\frac{\mathrm{T}}{2 \pi \mathrm{R}_{\mathrm{i}}^{2} \mathrm{hK}}-\frac{\tau_{0}}{\mathrm{~K}}\right)\left(1+\frac{\ln \left(\frac{\mathrm{T}}{2 \pi \mathrm{R}_{\mathrm{i}}^{2} \mathrm{~h} \tau_{0}}\right)}{1-\frac{\mathrm{T}}{2 \pi \mathrm{R}_{\mathrm{i}}^{2} \mathrm{~h} \tau_{0}}}\right)-\left(\frac{\mathrm{T}}{2 \pi \mathrm{R}_{\mathrm{o}}^{2} \mathrm{hK}}-\frac{\tau_{0}}{\mathrm{~K}}\right)\left(1+\frac{\ln \left(\frac{\mathrm{T}}{2 \pi \mathrm{R}_{\mathrm{o}}^{2} \mathrm{~h} \tau_{0}}\right)}{1-\frac{\mathrm{T}}{2 \pi \mathrm{R}_{\mathrm{o}}^{2} \mathrm{~h} \tau_{0}}}\right)=4 \pi \mathrm{N}
$$

or:

$$
\mathrm{T}=\frac{4 \pi \mathrm{h} \tau_{0}}{\left(\frac{1}{\mathrm{R}_{\mathrm{i}}^{2}}-\frac{1}{\mathrm{R}_{\mathrm{o}}^{2}}\right)} \ln \left(\frac{\mathrm{R}_{\mathrm{o}}}{\mathrm{R}_{\mathrm{i}}}\right)+\frac{\mathrm{K} 8 \pi^{2} \mathrm{~h}}{\left(\frac{1}{\mathrm{R}_{\mathrm{i}}^{2}}-\frac{1}{\mathrm{R}_{\mathrm{o}}^{2}}\right)} \mathrm{N} \equiv \mathrm{G}+\mathrm{HN}^{1}
$$

So:

$$
\tau_{0}=\frac{\mathrm{G}}{4 \pi \mathrm{h}}\left(\frac{1}{\mathrm{R}_{\mathrm{i}}^{2}}-\frac{1}{\mathrm{R}_{\mathrm{o}}^{2}}\right) \frac{1}{\ln \left(\mathrm{R}_{\mathrm{o}} / \mathrm{R}_{\mathrm{i}}\right)} \equiv \hat{\tau}_{0}
$$


and

$\mathrm{K}=\frac{\mathrm{H}}{8 \pi^{2} \mathrm{~h}}\left(\frac{1}{\mathrm{R}_{\mathrm{i}}^{2}}-\frac{1}{\mathrm{R}_{\mathrm{o}}^{2}}\right) \equiv \mu$

So, although equation (45) seemed to be, at least at first sight, different from the corresponding equation for a Bingham fluid (eq. (29)), equations (47) and (48) reveal that the same Bingham flow curve $\tau(\dot{\gamma})$ is achieved for a Herschel-Bulkley fluid with a unit flow index $(\mathrm{n}=1)$.

\subsection{Solution for a Herschel-Bulkley fluid $(n \neq 1)$}

The main problem for $\mathrm{n} \neq 1$ consists of finding an analytical approximation for $\hat{\phi}(\mathrm{z}, 1, \mathrm{a})$ when $\mathrm{a} \neq 1$.

However, up to now such approximation has not yet been found, so equation (42) could only be solved by numerical means. The search for an analytical approximation is still going on. A direction for this approximation is given by:

$\frac{\hat{\phi}\left(\mathrm{z}=\mathrm{z}_{\mathrm{i}}, 1, \mathrm{a}\right)}{\hat{\phi}\left(\mathrm{z}=\mathrm{z}_{\mathrm{i}}, 1,1\right)}=\zeta_{1, \mathrm{i}} \cdot \mathrm{a}^{\zeta_{2, \mathrm{i}}}\left(\mathrm{R}^{2} \geq 0.99\right)$

If now, the z-dependency of $\zeta_{1}$ and $\zeta_{2}$ could be found, $\hat{\phi}(\mathrm{z}, 1, \mathrm{a})$ could be calculated as:

$\hat{\phi}(z, 1, a)=\zeta_{1}(z) \cdot a^{\zeta_{2}(z)} \cdot \hat{\phi}(z, 1,1) \equiv-\zeta_{1}(z) \cdot a^{\zeta_{2}(z)} \cdot \frac{\ln (1-z)}{z}$

Further work will be done to define $\zeta_{1}(\mathrm{z})$ and $\zeta_{2}(\mathrm{z})$ in order to find an analytical approximation for for $\hat{\phi}(\mathrm{z}, 1, \mathrm{a})$ when $\mathrm{a} \neq 1$.

In the case it would not be possible to find such an analytical approximation, the potentialities of the Tikhonov regularization method [1] and/or the wavelette-vaguelette decomposition method [2] to describe the fundamental flow of cement-based materials (in general, and self-compacting concrete in particular) reigned by a Herschel-Bulkley behaviour, will be investigated.

\section{CONCLUSIONS}

The aim of the paper was to find out the possibility of solving the inverse Couette problem by means of the integration method, needed for the rheological description of Belgian SCC mixes reigned by a shear thickening or Herschel-bulkley behaviour. The following conclusions are based on preliminary results from an ongoing work and are thus not the final ones. Within these limitations, the following conclusions may be drawn:

- A general description of the shear stress $\tau$ and shear rate $\dot{\gamma}$ is given, based on the constitutive equation. Then, the derivation of the Reiner-Riwlin equation is made in order to illustrate the solution of the Couette inverse problem for a Bingham fluid.

- For the Herschel-Bulkley fluid, the $\mathrm{T}(\mathrm{N})$ start equation could be integrated. Unfortunately, the solution of this integral contained a transcendent, nonpolynomial 
function (LerchPhi). So the inverse Couette problem could only be solved for the special case of a flow index $n=1$. The result revealed that in this special case, indeed the same flow curve $\tau(\dot{\gamma})$ as for a Bingham fluid is achieved.

- In the case $n \neq 1$, the integrated start equation could not be further analysed due to the mentioned LerchPhi function. However, research on this topic is still continuing.

\section{REFERENCES}

[1] Yeow, Y., Ko, W. and Tang, P., "Solving the inverse problem of Couette viscometry by Tikhonov regularization", J. Rheol. 44(6) (2000) 1335-1351.

[2] Ancey, C., "Solving the Couette inverse problem using a wavelet-vaguelette decomposition", $J$. Rheol. 49(2) (2005) 441-460.

[3] Reiner, M., "Deformation and Flow - An Elementary Introduction to Theoretical Rheology", (H. K. Lewis \& Co. Ltd., London, 1949).

[4] Vermant, J., "Applied Rheology”, (K.U.Leuven/CIT/Applied Rheology and Polymer Processing, Leuven, 2006).

[5] Cauberg, N., Dieryck, V. and Desmyter J., "Rheology of self-compacting concrete - validation of empirical test methods", in: "SCC 2005", Proc. of the combined $2^{\text {nd }}$ North American Conf. on the Design and Use of Self-Consolidating Concrete and the $4^{\text {th }}$ Int. RILEM Sym. on Self-Compacting Concrete', Oct./Nov. 2005 (Hanley Wood, Chicago, 2005) 765-773.

[6] Wallevik, Ó., "Rheology of Coarse Particle Suspensions, such as Cement Paste, Mortar and Concrete", Rheology course, Limelette, 2004.

[7] Wallevik, Ó, "The Rheology of Fresh Concrete and its Application on Concrete with and without Silica Fume", (PhD dissertation, NTNU Trondheim, 1990) < only available in Norwegian>.

[8] Macosko, C.W., "Rheology: Principles, Measurements and Applications", (Wiley, Sussex, 1994).

[9] Wallevik, J.E., "Rheology of Particle Suspensions, Fresh Concrete, Mortar and Cement Paste with Various Types of Lignosulfonates", (PhD dissertation, NTNU Trondheim, 2003).

[10] Fletcher, C.A.J., "Computational Techniques for Fluid Dynamics (vol. I)", $2^{\text {nd }}$ Edn (SpringerVerlag, Berlin-Heidelberg, 1990).

[11] Malvern, L. E., "Introduction to the Mechanics of a Continuous Medium", (Prentice-Hall, Englewood Cliffs, 1969). 